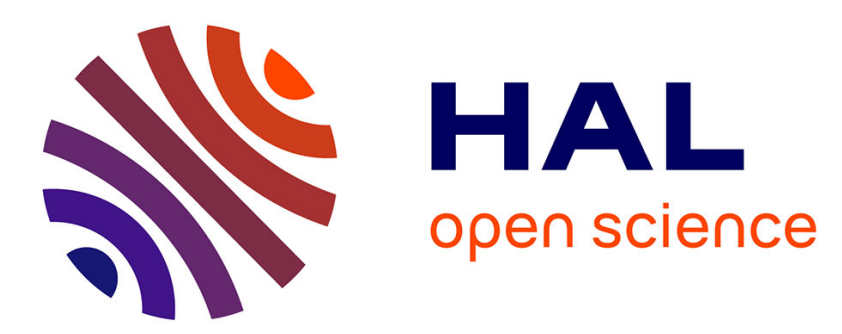

\title{
Online Identification and Visualization of the Statically Equivalent Serial Chain via Constrained Kalman Filter
} Alejandro González, Mitsuhiro Hayashibe, Philippe Fraisse

\section{To cite this version:}

Alejandro González, Mitsuhiro Hayashibe, Philippe Fraisse. Online Identification and Visualization of the Statically Equivalent Serial Chain via Constrained Kalman Filter. ICRA: International Conference on Robotics and Automation, May 2013, Karlsruhe, Germany. pp.5303-5308. lirmm-00830245

\section{HAL Id: lirmm-00830245 \\ https://hal-lirmm.ccsd.cnrs.fr/lirmm-00830245}

Submitted on 4 Jun 2013

HAL is a multi-disciplinary open access archive for the deposit and dissemination of scientific research documents, whether they are published or not. The documents may come from teaching and research institutions in France or abroad, or from public or private research centers.
L'archive ouverte pluridisciplinaire HAL, est destinée au dépôt et à la diffusion de documents scientifiques de niveau recherche, publiés ou non, émanant des établissements d'enseignement et de recherche français ou étrangers, des laboratoires publics ou privés. 


\section{Online Identification and Visualization of the Statically Equivalent Serial Chain via Constrained Kalman Filter}

\author{
Alejandro González
}

\author{
Mitsuhiro Hayashibe
}

\author{
Philippe Fraisse
}

\begin{abstract}
A human's center of mass (CoM) trajectory is useful to evaluate the dynamic stability during daily life activities such as walking and standing up. To estimate the subjectspecific CoM position in the home environment, we make use of a statically equivalent serial chain (SESC) developed with a portable measurement system. In this paper we implement a constrained Kalman filter to achieve an online estimation of the SESC parameters while accounting for the human body's bilateral symmetry. This results in constraining SESC parameters to be consistent with the human skeletal model used. The proposed identification method can inform the subject or the therapist, in real-time, about the quality of the on-going CoM estimation. This information can be helpful to reduce the identification time and establish a personalized protocol. A Kinect is used as a markerless motion capture system for measuring limb orientations while the Wii board is used to measure the subject's center of pressure $(\mathrm{CoP})$ during the identification phase. CoP measurements and Kinect data were recorded for four able-bodied subjects. The recorded data was then given to the proposed recursive algorithm to identify the parameters of the SESC online. A cross-validation test was performed to verify the identification performance. The results for these subjects are shown and discussed.
\end{abstract}

\section{INTRODUCTION}

It is expected that $16 \%$ of the world's population will become 65 years old or older by 2015 [1]. This fact underlines the importance of developing technologies to support the rehabilitation environment of elderly individuals. Monitoring systems are being developed to help diagnose a subject's mobility; such tools may be used to asses and prevent fall risks [2], providing additional tools for balance rehabilitation [3].

To avoid falling during unsupported standing, a human's center of mass (CoM) projection to the horizontal plane should be confined to the area delimited by his feet. The same is true for any other movement where small accelerations are applied to the CoM. Estimating CoM position for a human, or a humanoid robot, is important to evaluate postural stability. Additionally, CoM tracking can be used as a task during balance training, and to quantitatively evaluate a patient's improvement before, during and after following a rehabilitation program.

Currently, the most widely used method for CoM estimation makes use of information contained in tables, such as the ones provided by [4] or [5]; with it a weighted sum of each body segment's center of mass position is performed in order to determine the total CoM. This method requires

A. González, M. Hayashibe and P. Fraisse are with INRIA DEMAR Project and LIRMM, CNRS/University of Montpellier, France. \{gonzalezde, hayashibe, fraisse\}-at-lirmm.fr the position of each segment to be known. Usually, the segment's orientation is determined by a number of inertial sensors [6], [7] or by means of video analysis used to track a set of markers attached to the subject. This segment based approach to CoM estimation is dependent on the reliability of the anthropometric table, certain errors can occur if the subject does not match the population from which the table originated. That is, the anthropometric table describes the segment parameters for a specific population; the table's data will be different for subjects of different age, somatotype, and physical fitness level. Depending on the application, medical imaging techniques could also be used to improve the table estimates; however, such additional measurements increase the cost and the complexity of the estimation [8]. For example, the work done by Venture et al. [9] is capable of providing subject-specific mass and inertial parameters of each body segment, but as it requires accurate measurement of segment acceleration, a high-end motion capture system and force platform are necessary. Estimating the CoM position for a wide range of motions can also be done using the double integral method [10]. This method requires all movements to be performed on top of an instrumented surface. A subject-specific CoM estimation using the previously described methods is unsuitable for in-home rehabilitation due to high equipment costs.

The statically equivalent serial chain (SESC) method for CoM estimation of a linked chain can be used to overcome these difficulties. The SESC method was introduced by Espiau and Boulic [11] for the control of a tree shaped, linked structure. It was first applied by Cotton el al. to provide a personalized CoM estimation for a young subject [12], and later for a group of elderly patients [13]. The SESC method allowed them to estimate the subjects' CoM position but required the use of a marker based Motion Capture system. We have demonstrated that it is possible to use a low cost sensor like the Kinect to track CoM position with the SESC [14], making it attractive for in-home use.

In previous work [14]; we gathered all the necessary data before the identification process began. This batch style method gives no insight to the subject nor the therapist about the quality of the on-going identification. It is also likely to make the experimental time longer and have redundant postures for identification. In this paper, we aim at developing recursive algorithms which provide real-time information regarding the identification. In this way, a visual interface is provided for the user which can help to determine if the identification has converged or not. This knowledge offers a visual clue as to which limbs should be moved in 


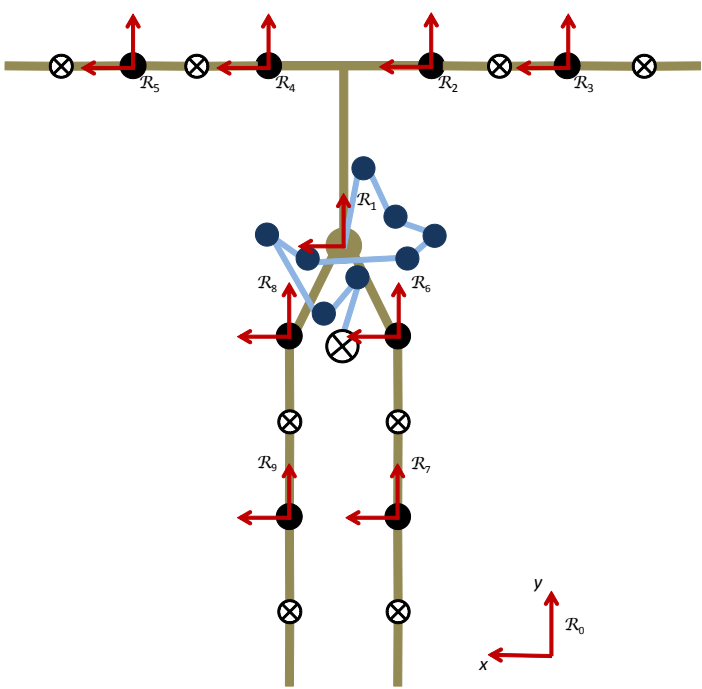

Fig. 1. The human skeleton model includes 9 links [17]. The SESC is depicted in blue.

order to improve the parameter estimate. Such framework can naturally allow the experimental procedure to be effective and minimize the measurement time for the identification [9]. In order to further optimize the identification, a constrained Kalman filter was used [15]. The filter allows knowledge of the mechanical model to be written into the state estimate, in many cases reducing the complexity of the system. Here we use it to introduce the assumption of the subject's bilateral symmetry. We expect the recursive approach to converge faster and with a minimum numbers of postures [16].

\section{Statically Equivalent Serial Chain model}

\section{A. Model definition}

A linked chain's CoM position can be expressed as a function of the chain's link orientations and a set of parameters determined by the chain's structure. These parameters can be thought of as containing the geometric description of the links of a virtual, open, serial chain whose end-effector is attached to the original chain's CoM. This chain is known as the statically equivalent serial chain (SESC) [11], [12].

In order to estimate the CoM position of a human subject we use skeleton model composed of 9 links connected by spherical joints (see Fig. 1). We consider this model to be a tree structure made up of four individual serial chains, all of them joined at the torso. To find the SESC parameters, the reference frame $\mathcal{R}_{i}$ is associated to each link. If the origin of $\mathcal{R}_{i}$ in frame $\mathcal{R}_{j}$ is given by the vector ${ }^{j} \vec{d}_{i}$, and the relative orientation between the frames is given by ${ }^{j} \mathbf{A}_{i}$, then the homogeneous transformation between them is given by (1).

$$
{ }^{j} \mathbf{T}_{i}=\left[\begin{array}{cc}
{ }^{j} \mathbf{A}_{i} & { }^{j} \vec{d}_{i} \\
\mathbf{0} & 1
\end{array}\right]
$$

Each link is assumed to have a mass $m_{i}$, located at a point $\vec{c}_{i}$ in its local reference frame. The total CoM position of the chain can be found by performing the matrix multiplication (2).

$$
\vec{C}_{M}=\left[\begin{array}{llll}
\mathbf{I} & \mathbf{A}_{1}^{*} & \ldots & \mathbf{A}_{9}^{*}
\end{array}\right]\left[\begin{array}{c}
0 \vec{d}_{1} \\
\vec{r}_{1} \\
\vdots \\
\vec{r}_{9}
\end{array}\right]
$$

where $\mathbf{I}$ is an identity matrix and $\mathbf{A}_{i}^{*}$ is the 3 -by-3 orientation matrix of link $i$ with respect to the global frame. The values in $\vec{r}_{i}$ describe the geometry of the SESC and can be explicitly determined as a function of the links' masses, their CoM position in the local reference frame, and the distance between the origins of two consecutive frames. For details on how to obtain $\vec{r}_{i}$, please refer to [11], [12], [14].

When all of the chain's joints are revolute or spherical, $\vec{r}_{i}$ is a constant valued 3-by-1 vector. Additionally, the CoM can be referenced to a floating frame attached to the skeleton. For example, by using the torso as a base for the SESC Eq. (2) can be rewritten as follows:

$$
{ }^{1} \vec{C}_{M}=\left[\begin{array}{lll}
\mathbf{A}_{1}^{*} & \ldots & \mathbf{A}_{9}^{*}
\end{array}\right]\left[\begin{array}{c}
\vec{r}_{1} \\
\vdots \\
\vec{r}_{9}
\end{array}\right]=\mathbf{B} \vec{R}
$$

where ${ }^{1} \vec{C}_{M}$ is the CoM position measured from the origin of $\mathcal{R}_{1}, \mathrm{~B}$ contains the orientation matrices for all of the chain's links, and $\vec{R}$ represents a 27 -by-1 vector of subject-specific parameters.

\section{B. Model reduction}

It was noted in [12] that due to the redundancy of the SESC, different $\vec{R}$ vectors may result in the same CoM estimate. We hope to obtain a parameter vector that is consistent with the physical model. To do this, we make the following assumptions to remove some of the chain's redundancies: i) The floating frame $\mathcal{R}_{1}$ is attached to the skeleton at the torso. ii) Bilateral symmetry exists, as we consider the left arm and leg to be identical in mass and size to the right ones. iii) As depicted in Fig. 1, one axis of the associated reference frame $\mathcal{R}_{i}$ lies on the line segment connecting two joints. iv) The CoM position for all limb segments, $\vec{c}_{i}$, also lies in this straight line. The last two assumptions result in some of the values in $\vec{R}$ to be equal to zero. These values and their corresponding columns in B can be removed without affecting the result. With these assumptions we decrease the number of unknown parameters from 27 to 11 constants. That is, $\vec{r}_{1}$ remains a 3-by-1 vector while $r_{2 \ldots 9}$ are represented by only 1 scalar each. In Sec. IIIB we use assumption ii) to further reduce the model by applying physical constraints to the estimated vector $\hat{\vec{R}}$.

\section{Parameter Estimation}

To determine the geometric parameters of an open ended serial chain such as the one described in Eq. (3), we may use a batch approach in which all data is collected first; or a recursive one where each new measurement improves our knowledge of $\hat{\vec{R}}$ [16]. To identify the SESC parameters the batch approach was used in [12], [14], but has the 
disadvantage of requiring a large number of postures to be performed by the subject as it offers no feedback as to which postures offer new information. To make the identification procedure more user-friendly, we implement the recursive approach. For either method, the same principles apply: i) A reasonable estimate of the CoM position for a set of calibration postures is needed. ii) A numerical estimation of $\vec{R}$ can be achieved without a complete knowledge of the subject's CoM position. In [12], it was noted that the use of partial information requires a larger number of data points to maintain accuracy. In this way, we define the measurement vector $\vec{Y}$ as:

$$
\vec{Y}=\left[\begin{array}{l}
\mathbf{B}_{x} \\
\mathbf{B}_{z}
\end{array}\right] \vec{R}
$$

CoP offers a good approximation for the ground plane projection of CoM during quiet standing due to the small accelerations experienced by the CoM [12]. To determine if a posture is stable enough, we observe the $\mathrm{CoP}$ position and the limbs' roll-pitch-yaw angles during a 30 sample window. This corresponds approximately to 1 second. We look at the standard deviation of the angles and $\mathrm{CoP}$ values to judge if the pose was stable during this time window to be used as identidification data.

\section{MODEL IDENTIFICATION}

\section{A. Kalman Filter}

The Kalman filter is utilized to estimate the value of a state vector evolving in a dynamical system. It can also be used to determine a set of constant values from a group of noisy measurements. Consider a linear dynamic system defined as:

$$
\vec{X}_{k}=\mathbf{F}_{k-1} \vec{X}_{k-1}+\vec{w}_{k}
$$

where the subindex $k$ denotes the time step. The evolution of the state vector $\vec{X}_{k}$ is dictated by the matrix $\mathbf{F}_{k-1}$ and a zero mean process noise $\vec{w}_{k}$ with covariance $\mathbf{Q}_{k}$. Vector $\vec{X}_{k}$ may be estimated based on a series of measurements $\vec{Y}_{k}$; where each measurement is a linear combination of the states and a zero mean measurement noise $\vec{v}_{k}$ with covariance $\mathbf{R}_{k}$. That is to say, each measurement can be expressed in the form:

$$
\vec{Y}_{k}=\mathbf{H}_{k} \vec{X}_{k}+\vec{v}_{k}
$$

where $\mathbf{H}_{k}$ is referred to as the configuration matrix.

When a recursive estimation method is applied, each new measurement is used to update the current state vector estimate $\hat{\vec{X}}_{k}$. The Kalman filter, provides an optimal linear solution to the problem where the noisy system defined by Eq. (5) and (6) [15].

If the state vector $\vec{X}$ is fixed $\left(\mathbf{F}_{k-1}=\mathbf{I}\right)$ and subject to noise, the Kalman filter equations may be written as follows [15], [16]:

$$
\begin{aligned}
\mathbf{P}_{k}^{-} & =\mathbf{P}_{k-1}^{+}+\mathbf{Q}_{k-1} \\
\mathbf{K}_{k} & =\mathbf{P}_{k}^{-} \mathbf{H}_{k}^{T}\left(\mathbf{H}_{k} \mathbf{P}_{k}^{-} \mathbf{H}_{k}^{T}+\mathbf{R}_{k}\right)^{-1} \\
\hat{\vec{X}}_{k} & =\hat{\vec{X}}_{k-1}+\mathbf{K}_{k}\left(\vec{Y}_{k}-\mathbf{H}_{k} \hat{\vec{X}}_{k-1}\right) \\
\mathbf{P}_{k}^{+} & =\left(\mathbf{I}-\mathbf{K}_{k} \mathbf{H}_{k}\right) \mathbf{P}_{k}^{-}
\end{aligned}
$$

where $\mathbf{P}_{k}^{-}$and $\mathbf{P}_{k}^{+}$represent the estimation covariance matrices before and after updating the state, $\mathbf{I}$ is an identity matrix and $\mathbf{K}_{k}$ is the optimal Kalman filter gain which minimizes the squared error of the estimation (rmse). In other words this gain minimizes the function $J_{k}=\left(\vec{X}-\hat{\vec{X}}_{k}\right)^{T}\left(\vec{X}-\hat{\vec{X}}_{k}\right)$. A level of robustness may be introduced by appropiately selecting the noise covariance $\mathbf{Q}_{k}$. It can be used to account for modeling errors by increasing the filter's response to new measurements [15].

When estimating a constant vector $\hat{\vec{X}}$ using recursive techniques, it is possible to determine, in real-time, which values have been identified. Convergence of the states may be observed in the diagonal values of $\mathbf{P}$, which decreases as stimulating data is processed. In other words, the values of $\mathbf{P}$ hint as to which postures should be performed to obtain additional information.

\section{B. The constrained Kalman filter}

The performance of a Kalman filter sometimes can be improved with additional knowledge of the model. All indepth knowledge of the system may be expressed as a set of linear and non linear equality and inequality constraints on the states, and the filter may be rewritten to express them.

Linear equality constraints may be implemented into the filter by either: i) Reducing the model by creating a simple one in which the new states are a linear combination of the original states. This can potentially increase computation speed. ii) Increasing the model by introducing perfect measurements. iii) Projecting the filter estimate into the acceptable state space. Linear inequality constraints can be dealt with using a quadratic programming approach which minimizes the distance between the constrained and unconstrained state estimates. In this way the constrained estimate is not taken into account to determine the updated state once a new measurement is available. It has been suggested that a better way to include linear inequality constraints, as well as nonlinear equality and inequality constraints into the model is to redefine the statistic distribution associated with the Kalman filter [15], [18]. The redefinition consists in truncating the probability distribution function of the filter in such a way as to include only the acceptable solutions for the state estimate.

It is possible to add a set of linear equality constraints to our model by recognizing the subject's bilateral symmetry, as stated in Sec. II-B. After explicitly developing the relations for each SESC parameter we note that: $\vec{r}_{2}=-\vec{r}_{4}, \vec{r}_{3}=-\vec{r}_{5}$, $\vec{r}_{6}=\vec{r}_{8}$, and $\vec{r}_{7}=\vec{r}_{9}$. The corresponding columns of $\mathbf{B}$ (3) may be combined as needed to reflect these constraints [15]. The new, constrained model used for identification is given by:

$$
\begin{aligned}
\vec{R}_{k} & =\vec{R}_{k-1}=\vec{R} \\
\vec{Y}_{k} & =\mathbf{B}_{k} \vec{R}_{k}+\vec{v}_{k}
\end{aligned}
$$

where $\vec{R}$ has been reduced to a 7-by-1 vector which contains the SESC parameters, $\vec{Y}_{k}$ is the 2-by-1 position of the CoP as measured from $\mathcal{R}_{1}$ at time $k$, and $\mathbf{B}_{k}$ is the 2-by-7 
configuration matrix of the system which can be obtained by measuring the orientation of the limbs.

\section{SENSORS}

\section{A. Kinect}

The Kinect sensor was originally conceived as an input device to be used with video games. This sensor can estimate the depth of a scene by projecting a pattern of infrared light and interpreting the deformed pattern observed by a camera mounted on the device [2]. Using the middleware provided by OpenNi-PrimeSense a skeleton can be fitted over any person entering the scene. Joint positions and limb orientations may be tracked, provided that the subject remains mostly unoccluded and inside the field of vision of the sensor.

\section{B. Wii board}

The Wii board was also intended to function as a video game input device. It is capable of providing the vertical component of the ground reaction force and the CoP position of the subject. As it functions similarly to a force platform, the player can interact with a virtual environment by shifting his weight and moving his CoM along different trajectories. This functionality has attracted the attention of the medical community as it can be used as an inexpensive tool for the rehabilitation of stroke patients [3]. Communication with the board is achieved via Bluetooth. For this purpose we make use of the open source wiiuse project.

\section{EXPERIMENTAL RESULTS}

\section{A. Experimental setup and Data collection}

Four able-bodied subjects, denoted as Sb01-Sb04, were asked to stand on top of a Wii board. They were then required to perform and hold a series of different postures in order to calibrate their individual SESC. Although a set of postures was suggested to the subjects, no restrictions were given regarding exact limb orientation. All movements performed in the 3D space were dependent on the subject's physical capacities. Fig. 2 contains a series of frames from the Kinect recording and shows the test setup.

CoP information from the Wii board and skeletal information from the Kinect were simultaneously recorded to be used during the development of the recursive identification algorithm. The Kinect camera was placed facing the subject, three meters away from him. A nominal sampling rate of $30 \mathrm{~Hz}$ has been reported while using the Kinect. We have found that this frequency is dependent on the system on which the middleware runs. For our tests, in average, a frame rate of $24 \mathrm{~Hz}$ was achieved. In order to be used for identification, $\mathrm{CoP}$ data was expressed on the global reference frame, that of the Kinect. Two recordings were made for each subject; one for identification purposes, and one for cross-validation.

\section{B. Model Identification and Cross-validation}

During identification, angular and CoP data were observed in a 30 frame window. To determine if the information was suitable for identification, we observed the standard deviation of the 27 roll-pitch-yaw orientation angles and the twodimensional CoP position. If the sum of the deviations in the limbs' orientation angles was limited to be $40 \mathrm{deg}$ and the sum of the CoP deviations was under $13 \mathrm{~mm}$, then the posture performed during that window was considered static and was used for identification.

The SESC parameter identification was done recursively using the constrained Kalman filter described previously. One recording session was used for identification while a different recording was used for cross-validation to evaluate the results. Fig. 3 shows the ground projection of the estimated CoM position and the measured CoP position during the cross-validation test of Sb03. Fig. 4 shows the evolution of the estimated model parameters. In this figure, it is possible to observe the parameter convergence.

Table I shows the identified SESC parameters $\vec{R}$ for all four subjects, as well as the estimation covariance $\mathbf{P}_{j j}$ of each parameter. The root mean squared error ( $r m s e)$ obtained during the cross-validation test is also shown. In Fig. 5 we show the faster convergence of the SESC parameters when the constrained filter is used. For clarity, we show only the parameters linked to the upper arm for Sb03. The constrained estimate, $\vec{r}_{4}$, is seen to converge to a steady value faster than the unconstrained version of $\vec{r}_{4}^{*}$ and $\vec{r}_{6}^{*}$ when using the same measurements.

\section{DISCUSSION AND FUTURE WORK}

Even though the model was augmented from the one presented in [14] to include full 3D motion, the number of parameters for the model description was only doubled, not tripled as expected. This small increase in the number of required parameters was mainly due to a careful model definition and the commonly made assumptions described. This is convenient since the number of postures required for the geometric calibration depends on the number of parameters to identify. It is noted that the SESC parameters corresponding to the arms, $\vec{r}_{2,3}$, were not entirely consistent with the model definition. Specifically, due to the frame definition both parameters should have a negative value. The estimated values may be due to the small masses of these segments making them difficult to be identified. The estimation of $\vec{r}_{2}$ and $\vec{r}_{3}$ may be improved by the addition of the corresponding inequality constraints.

During the recording sessions and by observing the evolution of the parameter estimates while testing, it was possible to obtain some insight into which poses can give valuable information to the filter. This finding can be used in the design of a sequence of postures for identification. As the current sequence is not determined with such consideration, it implies the possibility to improve the feeding data's excitability regarding the parameters. It can result in further optimizing the whole identification protocol. For example, the parameter convergence that can be observed in Fig. 4 

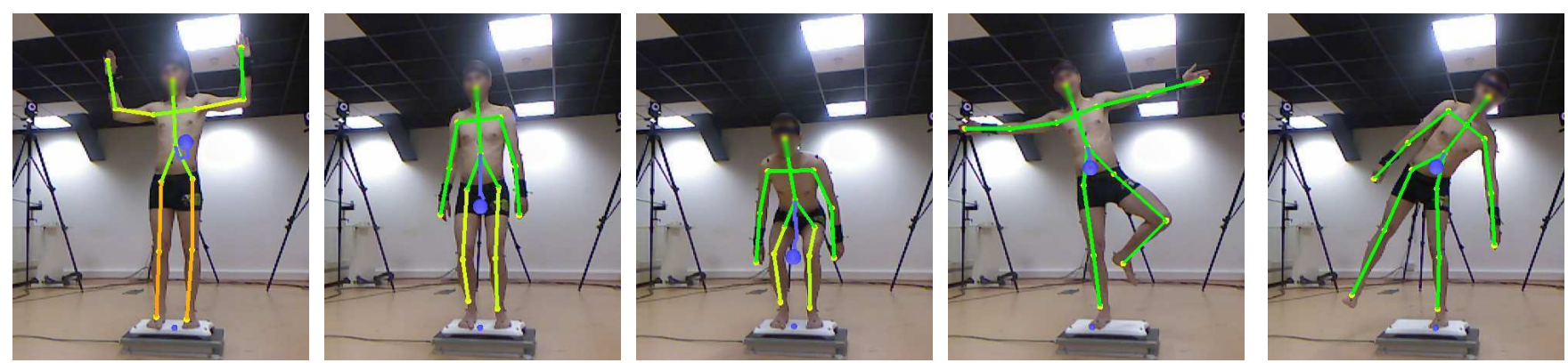

Fig. 2. Online statically equivalent serial chain (SESC) parameter estimation. The length of each of the SESC's link is updated when a static pose has been found and can be observed in real-time. The color of the skeleton can be updated so as to inform to the subject and/or therapist about the quality of the indentification. In the figure, limbs starting out in red, gradually turn green as their corresponding parameters converge.
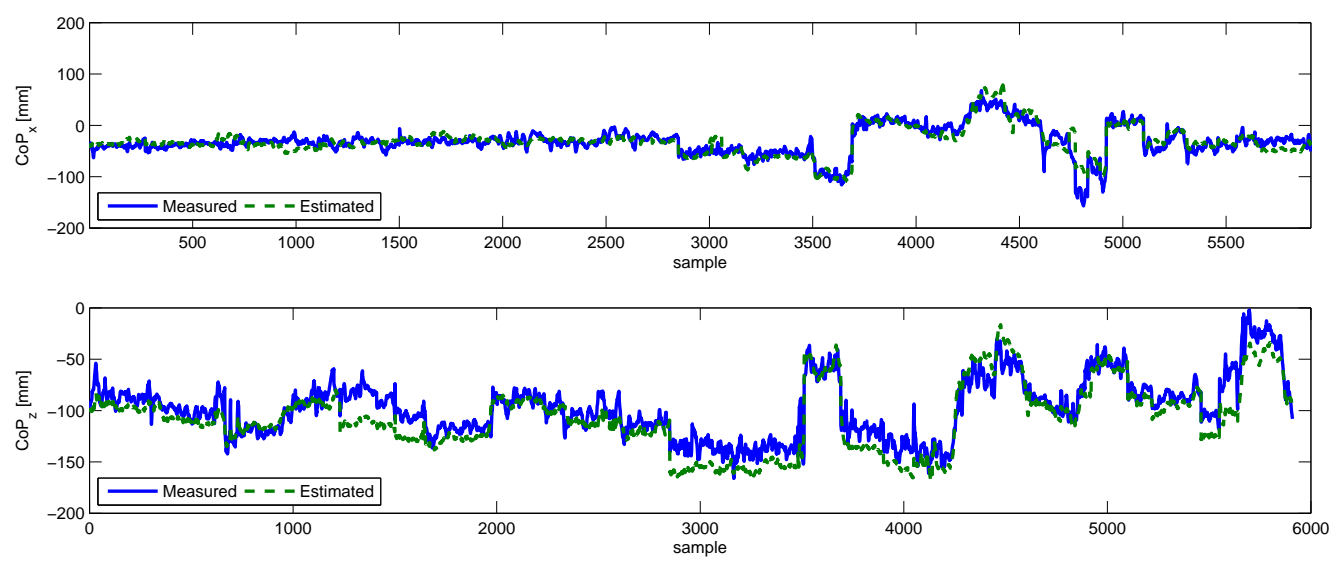

Fig. 3. Cross validation results for Sb03. Measured CoP, shown in blue, is compared to the ground projection of the estimated subject's CoM shown with a dotted green line.

TABLE I

RESULTS OF THE PARAMETER ESTIMATION.

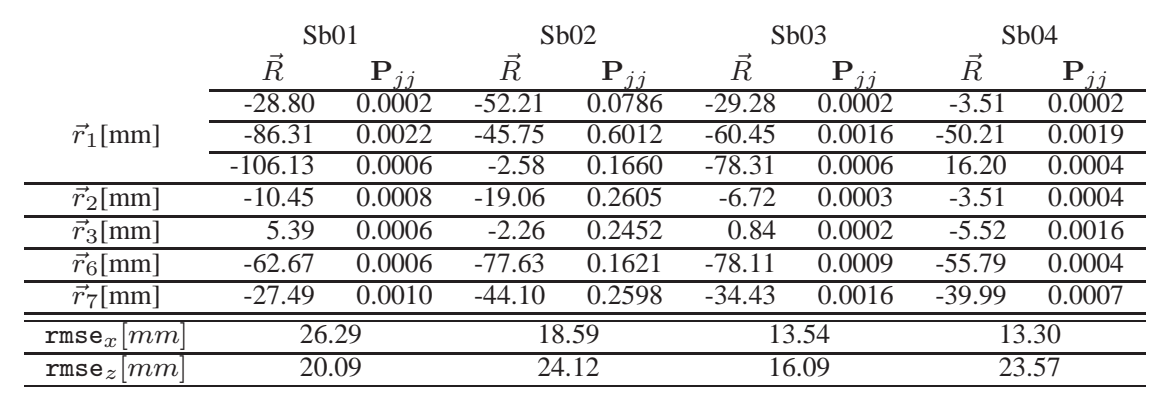

shows that the arms were adequately characterized early in session while the torso and leg segments approached their final value closer to the end of the trial. This is consistent with the sequence of postures performed by the subject which began by arm movements. Once the arm parameters converged, postures which engage the lower extremities should have been performed to make an efficient identification. The set of postures used for identification can be seen in the joint video. So far only able-bodied, young subjects have been asked to participate in the study. Some of the postures used for the SESC calibration should not be performed unassisted by individuals with balance disorders as they are too physically demanding and may present a fall risk. Our current work is focused on finding an ideal identification strategy to include these individuals. A new strategy may include a multicontact situation, common in rehabilitation, designed to assist the subject to maintain balance during the identification.

\section{CONCLUSION}

In this paper, we analyzed the feasibility of identifying SESC parameters using a recursive algorithm such as the Kalman filter. In addition, constraints can be included into the estimation in order to obtain reliable parameters which are consistent with the definition of the model and the physical characteristics of the subject. It was encouraging to note in Table I that the subjects' parameters are roughly proportional 


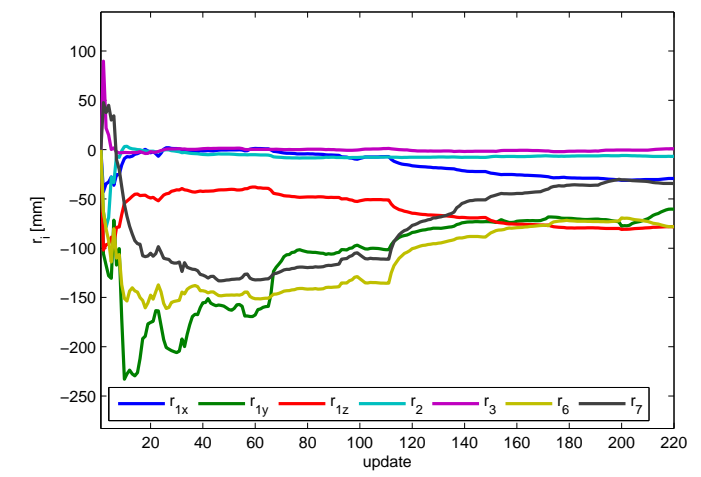

Fig. 4. Estimated values of the SESC parameters after each update.

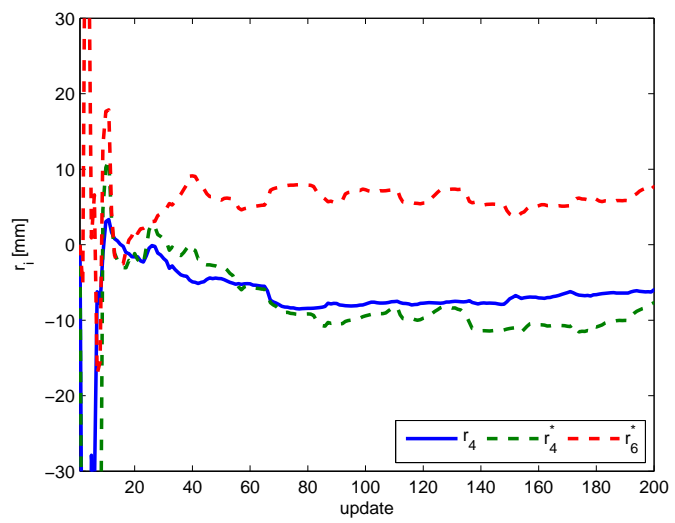

Fig. 5. Parameters of the constrained Kalman, $\vec{r}_{i}$, filter converges faster than the unconstrained version, $\vec{r}_{i}^{*}$, when the same data is given to both filters during identification.

to each individual's height. We have achieved good results with this identification obtaining a rmse as low as $15 \mathrm{~mm}$ for Sb03 while tracking the CoM ground projection with the Kinect camera placed 3 meters away from the subject.

We focused on showing that the geometric calibration of the SESC can be done online by using the recursive techniques. This is an important improvement over the previously used batch approach since the subject can perform the postures with visual feedback. He can stop performing postures just after the computation has converged, all the while checking the resulting CoM estimation. As seen in Fig. 2, CoM estimate approaches the subject's body center during identification. Also, the coloring of the segment to inform the subject about which part of his body should be moved to promote convergence. The framework where the subject can adaptively give effective postures to the adaptive filter makes the identification closer to the optimal.

It is important to note that after the SESC parameter identification, subject-specific CoM position can be estimated only from Kinect information, even without a Wii board present. This makes it appropriate for rehabilitation purposes inside the in-home environment [19]. In addition, it is also important to emphasize that postures given during identification were necessarily "static" as we used a CoP sensor; but after the identification, the estimated CoM by the SESC is valid and expected to be accurate also for "dynamic" motions as long as a good limb orientation can be measured.

\section{ACKNOWLEDGMENT}

This work is supported by Agence Nationale de la Recherche (ANR) under grant ANR-09-SEGI-011 in the context of the R2A2 project.

\section{REFERENCES}

[1] United Nations, "World Population Ageing," Population Division of the Department of Economic and Social Affairs of the United Nations. Population Division of the Department of Economic and Social Affairs of the United Nations, 2009.

[2] E. E. Stone and M. Skubik, "Evaluation of an inexpensive depth camera for passive in-home fall risk assessment," Pervasive Computing Technologies for Healthcare, pp. 71-77, 2011.

[3] M. W. Kennedy, J. P. Schmiedeler, C. R. Crowell, M. Villano, A. D. Striegel, and J. Kuitse, "Enhanced feedback in balance rehabilitation using the Nintendo Wii Balance Board," in 2011 IEEE 13th International Conference on e-Health Networking, Applications and Services. IEEE, Jun. 2011, pp. 162-168.

[4] D. A. Winter, Biomechanics and motor control of human movement. Hoboken, N.J.: John Wiley \& Sons, Inc, 2005.

[5] P. de Leva, "Adjustments to Zatsiorsky-Seluyanov's segment inertia parameters," Journal of Biomechanics, vol. 29, no. 9, pp. 1223-1230, Sep. 1996.

[6] A. P. L. Bó, M. Hayashibe, and P. Poignet, "Joint angle estimation in rehabilitation with inertial sensors and its integration with Kinect." Engineering in Medicine and Biology, vol. 2011, pp. 3479-83, Jan. 2011.

[7] V. Bonnet, C. Mazzà, P. Fraisse, and A. Cappozzo, "A least-squares identification algorithm for estimating squat exercise mechanics using a single inertial measurement unit." Journal of biomechanics, Mar. 2012.

[8] M. A. Jaffrey, "Estimating Centre of Mass Trajectory and SubjectSpecific Body Segment Parameters Using Optimisation Approaches," $\mathrm{PhD}$, Victoria University, 2008.

[9] G. Venture, K. Ayusawa, and Y. Nakamura, "Real-time identification and visualization of human segment parameters." Аnnual International Conference of the IEEE Engineering in Medicine and Biology Society, vol. 2009, pp. 3983-6, Jan. 2009.

[10] H. M. Schepers, E. H. F. van Asseldonk, J. H. Buurke, and P. H. Veltink, "Ambulatory estimation of center of mass displacement during walking." IEEE transactions on bio-medical engineering, vol. 56, no. 4, pp. 1189-95, Apr. 2009.

[11] B. Espiau and R. Boulic, "On the Computation and control of the mass center of articulated chains," Research Report, no. 3479, 1998.

[12] S. Cotton, A. Murray, and P. Fraisse, "Estimation of the Center of Mass: From Humanoid Robots to Human Beings," IEEE/ASME Transactions on Mechatronics, vol. 14, no. 6, pp. 707-712, Dec. 2009.

[13] S. Cotton, M. Vanoncini, P. Fraisse, N. Ramdani, E. Demircan, A. P. Murray, and T. Keller, "Estimation of the centre of mass from motion capture and force plate recordings : a study on the elderly." Applied Bionics and Biomechanics, vol. 8, no. 2, pp. 67-84, 2011.

[14] A. González, M. Hayashibe, and P. Fraisse, "Estimation of the Center of Mass with Kinect and Wii balance board," in EEE/RSJ International Conference on Intelligent Robots and Systems, 2012, pp. 1023-1028.

[15] D. Simon, Optimal State Estimation: Kalman H Infinity, and Non Linear Approaches. John Wiley \& Sons, Inc, 2006.

[16] B. W. Mooring, Z. S. Roth, and M. R. Driels, Fundamentals of Manipulator Calibration. Wiley Press, 1991.

[17] PrimeSense, "PrimeSense Nite Algorithms 1.5," 2011.

[18] S. J. Julier and J. J. LaViola, Jr., "On Kalman Filtering With Nonlinear Equality Constraints," IEEE Transactions on Signal Processing, vol. 55, no. 6, pp. 2774-2784, Jun. 2007.

[19] A. González, M. Hayashibe, and P. Fraisse, "Subject-specific Center of Mass Estimation for In-home Rehabilitation - Kinect-Wii board vs. Vicon-Force plate," in International Conference on NeuroRehabilitation, J. L. Pons and D. Torricelli, Eds., vol. 2. Toledo, Spain: Springer, 2012, pp. 705-709. 